\title{
Role of TLR4/NF-kB Signalling Pathway in Pulmonary Arterial Hypertension in Patients with Chronic Obstructive Pulmonary Disease
}

\author{
Zhi Tong Zuo ${ }^{1}$, Ya Ma ${ }^{2}$, Yan Sun ${ }^{1}$, Cui Qing Bai ${ }^{1}$, Hai Yue Zhou ${ }^{1}$ and Bao Hua Chen ${ }^{1}$ \\ ${ }^{1}$ Department of Respiratory Disease, The Hospital Affiliated to Jiangnan University, Wuxi, Jiangsu, China \\ ${ }^{2}$ Wuxi Medical College of Jiangnan University, Wuxi, Jiangsu, China
}

\begin{abstract}
Objective: To determine the role of toll-like receptor 4 (TLR4)/ nuclear factor kappa B (NF-KB) signalling pathway in pulmonary arterial hypertension (PAH) in patients with chronic obstructive pulmonary disease (COPD).

Study Design: An experimental study.

Place and Duration of Study: Department of Respiratory Disease, The Hospital Affiliated to Jiangnan University, Wuxi, Jiangsu, China, from June 2018 to December 2019.

Methodology: Subjects included 98 COPD patients and 22 healthy individuals (control group). COPD patients were divided into two groups as PAH group (PAH group, $n=57$ ) and normal pulmonary arterial pressure group (nPAP group, $n=41$ ). TLR4 and NF-KB in peripheral blood mononuclear cells (PBMC) were measured by real-time polymerase chain reaction (RT-PCR); and inflammatory cytokine of IL- 6 and TNF- $\alpha$ were estimated by enzyme-linked immunosorbent assay (ELISA) of three groups.

Results: The levels of TLR4, NF-KB and inflammatory cytokine of IL- 6 and TNF- $\alpha$ of PAH group were higher than those in nPAP group and controls (all $p<0.05$ ); and controls had a lower levels of TLR4, NF-KB and TNF- $\alpha$ than those $n$ PAP group patients (all $p<0.05$ ) except for PAP and IL-6 ( $p=0.121$ and $p=0.304$, respectively). The expression levels of TLR4 and NF-KB in PBMC were positively related to that PAP and inflammatory cytokine of IL- 6 and TNF- $\alpha$ in PAH patients with COPD (all $p<0.05$ ), but the positive correlation betweenIL- 6 and TNF- $\alpha$ expression level was not established $(p=0.170)$. All parameters in the nPAP group had no significant correlation to each other, it is the same in control (all $p>0.05$ ).

Conclusions: Inflammatory mechanisms play an important role in the development of PAH in patients with COPD. TLR4/NF-KB signal transduction pathway is involved in the pathogenesis of PAH, and the expression levels of TLR4/NF-KB may reflect the severity of PAH in patients with COPD.
\end{abstract}

Key Words: Toll-like receptor 4, Nuclear factor kappa B, Pulmonary arterial hypertension, Chronic obstructive pulmonary disease, Inflammatory cytokine.

How to cite this article: Zuo ZT, Ma Y, Sun Y, Bai CQ, Zhou HY, Chen BH. Role of TLR4/NF-kB Signalling Pathway in Pulmonary Arterial Hypertension in Patients with Chronic Obstructive Pulmonary Disease. J Coll Physicians Surg Pak 2020; 30(06):568-573 https://doi.org/10.29271/jcpsp.2020.06.568.

\section{INTRODUCTION}

Toll-like receptor 4 / nuclear factor kappa B (TLR4/NF-KB) is a signal pathway which is closely related to inflammation. ${ }^{1}$ The study showed that the appearance of pulmonary arterial hypertension (PAH) was accompanied with inflammation of pulmonary artery endothelial cells.

Correspondence to: Zhi Tong Zuo, Department of Respiratory Disease, The Hospital Affiliated to Jiangnan University, 585 Xingyuan North Road, Wuxi, 214041, Jiangsu, China

E-mail: zzt555@163.com

Received: March 23, 2020; Revised: June 17, 2020;

Accepted: June 24, 2020

DOI: https://doi.org/10.29271/jcpsp.2020.06.568
The levels of inflammatory cytokines in PAH patients were significantly increased and the inflammatory factors were regulated by NF-KB. ${ }^{2}$ As the upstream receptor of NF-KB, the activation of toll-like receptors (TLRs) leads to the expression of a large number of NF-kB, then they initiate and regulate the expression of a series of inflammatory cytokines which are involved in the inflammatory response. As the most important member of the TLR family, TLR4 plays an important role in promoting the synthesis and release of cytokines and inflammation, affects the immune response and so on. ${ }^{3}$

The objective of this study is to detect the expression levels of TLR4, NF-KB in peripheral blood mononuclear cells and inflammatory factors in serum of chronic obstructive pulmonary disease (COPD) patients and healthy subjects, to explore the role of TLR4/NF-KB pathway in the formation process of pulmonary hypertension in COPD patients. 
Table I: Regression analysis of COPD with PAH patients.

\begin{tabular}{|c|c|c|c|c|}
\hline Correlation & Regression equation & $r$ & $t$ & $p$ \\
\hline PAP and TLR4 & $y=1.464+0.024 x$ & 0.439 & 3.619 & 0.001 \\
\hline PAP and NF-KB & $y=-0.520+0.056 x$ & 0.738 & 8.117 & $<0.001$ \\
\hline PAP and IL-6 & $y=1.679+0.027 x$ & 0.439 & 3.624 & 0.001 \\
\hline PAP and TNF- $\alpha$ & $y=1.299+0.028 x$ & 0.452 & 3.757 & $<0.001$ \\
\hline TLR4 and NF-kB & $y=2.066+0.311 x$ & 0.432 & 3.554 & 0.001 \\
\hline TLR4 and IL-6 & $y=1.645+0.409 x$ & 0.462 & 3.862 & $<0.001$ \\
\hline TLR4 and TNF- $\alpha$ & $y=2.167+0.285 x$ & 0.326 & 2.555 & 0.013 \\
\hline NF-kB and IL-6 & $y=1.670+0.452 x$ & 0.367 & 2.928 & 0.005 \\
\hline NF-KB and TNF- $\alpha$ & $y=1.829+0.445 x$ & 0.367 & 2.922 & 0.005 \\
\hline IL- 6 and TNF- $\alpha$ & $y=2.921+0.182 x$ & 0.184 & 1.391 & 0.170 \\
\hline
\end{tabular}

Table II: Regression analysis of nPAP group and the control group.

\begin{tabular}{|l|c|c|c|c|c|c|}
\hline \multirow{2}{*}{ Correlation } & \multicolumn{2}{|c|}{ nPAP group } & \multicolumn{2}{c|}{ Control group } \\
\cline { 2 - 7 } & $\boldsymbol{\beta}$ & $\mathbf{t}$ & $\mathbf{p}$ & $\boldsymbol{\beta}$ & $\mathbf{t}$ & \multicolumn{1}{c|}{$\mathbf{p}$} \\
\hline PAP and TLR4 & -0.295 & -1.925 & 0.062 & 0.102 & 0.459 & 0.651 \\
\hline PAP and NF-KB & 0.098 & 0.617 & 0.541 & 0.240 & 1.104 & 0.283 \\
\hline PAP and IL-6 & -0.121 & -0.764 & 0.450 & -0.289 & -1.349 & 0.192 \\
\hline PAP and TNF- $\alpha$ & 0.087 & 0.547 & 0.587 & 0.337 & 1.600 & 0.125 \\
\hline TLR4 and NF-KB & 0.009 & 0.056 & 0.955 & -0.020 & -0.088 & 0.931 \\
\hline TLR4 and IL-6 & 0.268 & 1.735 & 0.091 & 0.257 & 1.187 & 0.249 \\
\hline TLR4 and TNF- $\alpha$ & 0.266 & 1.722 & 0.093 & 0.073 & 0.326 & 0.748 \\
\hline NF-KB and IL-6 & -0.056 & -0.349 & 0.729 & -0.077 & -0.344 & 0.735 \\
\hline NF-KB and TNF- $\alpha$ & 0.115 & 0.725 & 0.473 & 0.335 & 1.591 & 0.127 \\
\hline IL-6 and TNF- $\alpha$ & -0.004 & -0.026 & 0.980 & -0.385 & -1.867 & 0.077 \\
\hline
\end{tabular}

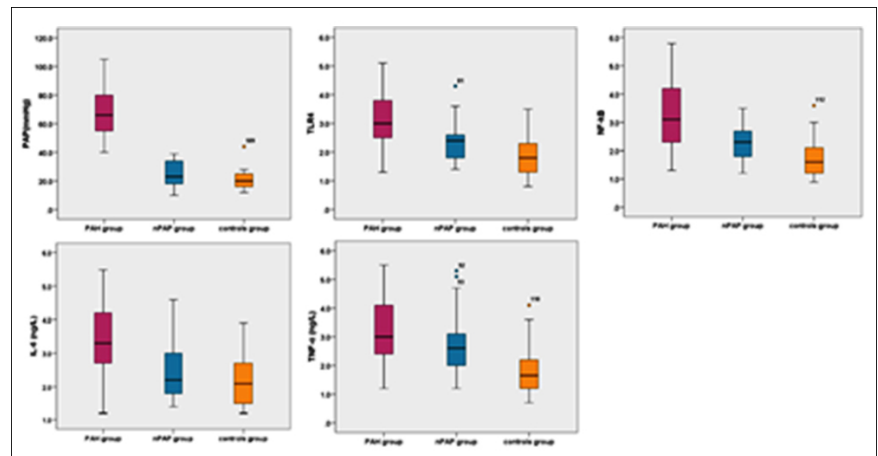

Figure 1: Comparison of PAP and the expression levels of TLR4 mRNA, NF-KB mRNA in PBMC and IL-6, TNF- $\alpha$ in serum in the three groups.

\section{METHODOLOGY}

Patients and controls were recruited from The Hospital Affiliated to Jiangnan University from June 2018 to December 2019. Subjects were 98 patients with COPD and 22 healthy individuals (control group). The pulmonary artery systolic pressure (PAP) was measured by Doppler ultrasound including the COPD patients, which were admitted to hospital and the healthy individuals. When the pulmonary artery systolic pressure was greater than or equal to 40 $\mathrm{mmHg}$, it was labelled with PAH. According to pulmonary artery systolic pressure, COPD patients were divided into two groups: PAH group ( $n=57,58.2 \%)$ and normal pulmo- nary arterial pressure group (n PAP group, $n=41,41.8 \%$ ). All patients met the diagnostic criteria of COPD. ${ }^{4}$ Subjects with evidence of other chronic respiratory diseases, serious liver, kidney, endocrine, coronary artery diseases, valvular heart disease, systemic hypertension or primary myocardial disease were excluded from the study. Informed consent for sample test was obtained. The study was approved by the Ethics Committee of The Affiliated Hospital of Jiangnan University.

Cardiac Doppler ultrasound examinations for four chambers of heart transection scan were done using specialised cardiac probe with 2.0 to $2.5 \mathrm{MHz}$ frequency. Backflow pressure of tricuspid valve was added to the right atrium pressure as the pulmonary artery systolic pressure.

Three $\mathrm{mL}$ of blood collection taken from the fasting elbow vein of the subjects, as transferred into ethylene diamine tetraacetic acid (EDTA) anticoagulant tube, centrifuged at $3000 \mathrm{r} /$ minute for 10 minutes. The supernatant were collected and tested using enzyme-linked immunosorbent assay (ELISA), according to the protocol of ELISA reagent kits. The ELISA kits of interleukin-6 (IL-6) and tumor necrosis factor- $\alpha$ (TNF- $\alpha$ ) were bought from United States $R \& D$ Systems Company. 


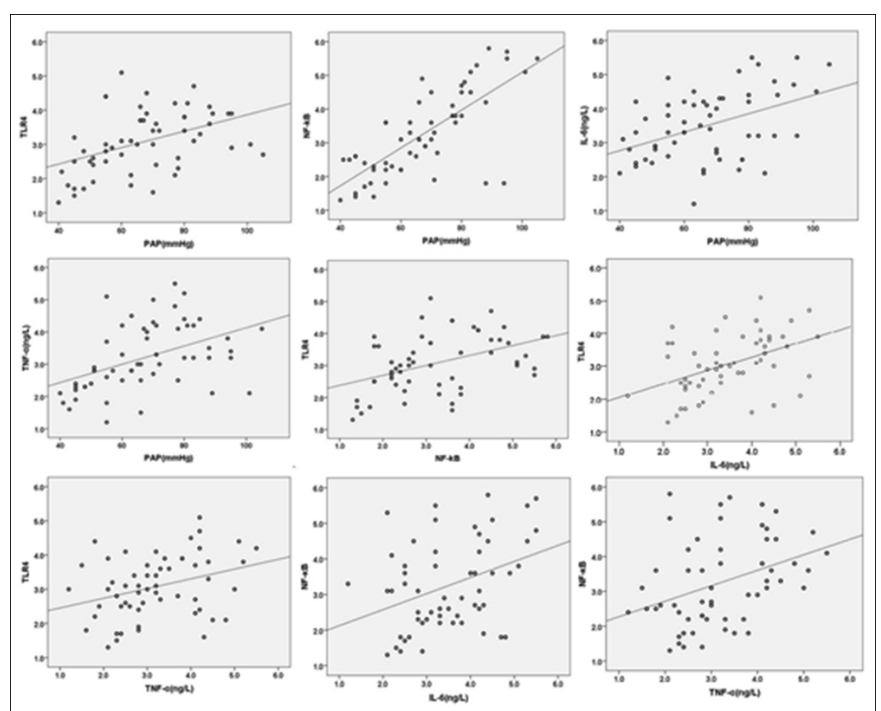

Figure 2: Regression analysis of PAH patients with COPD.

The operation / separation of peripheral blood monocytes was performed in strict accordance with the specification of lymphocyte separation fluid kit (Cedarlane, Canada). Total RNAs of TLR4 and NF-KB of PBMC were extracted with TRIzol reagent (American Invitrogen Company) by One Step Real-time polymerase chain reaction ( $R T-P C R$ ). With the template of the mRNA in the total RNA, c DNA was synthesised by reverse transcription (kit purchased from TAKARA Company), and $\beta$-actin as a reference. The PCR reaction condition was used: after pre-denaturation at $94^{\circ} \mathrm{C}$ for $5 \mathrm{~min}$, the amplification was done at 30 cycles, each cycle for $40 \mathrm{sec}$ at $94^{\circ} \mathrm{C}$, for $40 \mathrm{sec}$ at $50^{\circ} \mathrm{C}$, and for $30 \mathrm{sec}$ at $72^{\circ} \mathrm{C}$. After the cycle, the extension was done for $5 \mathrm{~min}$ at $72^{\circ} \mathrm{C}$ and then for $5 \mathrm{~min}$ at $4^{\circ} \mathrm{C}$. Primers were designed and synthesised by Shanghai Xiang Heng Biological Technology Co. Ltd. The specific primers is: TLR4 forward: 5'-GGTGGA AGTTGAACGAAT-3', reverse: 5'-AGATGATACCGCACG AC-3' NF-KB forward: 5'-AGAGCTAATCCGCCAAGC- 3', reverse: $5^{\prime}$-ACCGCCGAAACTATCCGAA-3'. Put the PCR products $8 \mu \mathrm{l}$ in $2 \%$ agarose gel (containing ethidium bromide $0.5 \mu \mathrm{g} / \mathrm{ml}$ ) and run electrophoresis at $120 \mathrm{~V}$ for $30 \mathrm{~min}$. Took pictures with $\mathrm{Gel}$ imaging system. Optical density ratio of $\beta$-actin and TLR4 was calculated as the relative expression levels of TLR4 mRNA and NF-kB mRNA.

The results were expressed as mean \pm SD. Frequencies were expressed as percentages and compared by using Chi-square test. Two independent samples were compared using the independent sample t-test. The normality of continuous variables was assessed using Shapiro-Wilk's W-test. One-Way ANOVA was used for analysing more than two groups of sample mean difference test. Pearson's correlation and linear regression analysis were used to determine the linear relationship between parameters. SPSS 22.0 and Graphpad 5.0 were adopted for calculation. All tests were two-tailed. The $p$-value less than 0.05 was considered to be statistically significant.

\section{RESULTS}

Among the $57 \mathrm{PAH}$ patients, there were 21 females $(36.8 \%)$ and 36 males (63.2\%). They aged between 52 and 97, with the mean age of $71.12 \pm 10.73$ years. Their COPD duration was from 6 years to 46 years. The $41 \mathrm{n}$ PAP group included 16 females (39.0\%) and 25 males (61.0\%), aged between 48 and 88 , with mean age of $68.71 \pm 12.21$ years. Their COPD duration was from 5 years to 30 years. In the 22 controls, there were 8 females (36.4\%) and 14 males (63.6\%), aged between 61 and 93 , with mean age of $71.68 \pm 8.59$ years. Difference in the gender and age of the three groups were not statistically significant, $(p=0.969$ and $p=0.466)$ respectively.

The mean measurements were PAP of PAH patients was $(67.21 \pm 16.39) \mathrm{mmHg}(24.66 \pm 8.77) \mathrm{mmHG}$ in nPAP group, and $(21.23 \pm 7.21) \mathrm{mmHG}$ in controls. As shown in Figure 1, increasing mRNA levels of TLR4 (PAH = 3.08 \pm 0.90 , $\mathrm{nPAP}=2.32 \pm 0.64$ and control $=1.86 \pm 0.67), \mathrm{NF}-\mathrm{kB}(\mathrm{PAH}=3.25$ $\pm 1.25, \mathrm{n} P A P=2.32 \pm 0.62$ and control $=1.77 \pm 0.68)$ in $\mathrm{PBMC}$ and serum levels of IL- $6(\mathrm{PAH}=3.50 \pm 1.01, \mathrm{nPAP}=2.49 \pm 0.88$ and control=2.26 \pm 0.76$)$ were seen. TNF- $\alpha(P A H=3.20$ $\pm 1.03, \mathrm{nPAP}=2.67 \pm 0.96$ and control $=1.91 \pm 0.95$ ) were also elevated in PAH patients with COPD when compared with nPAP group patients and controls. All $p<0.001$. Similarly, the levels of TLR4 NF-KB and TNF- $\alpha$ were also elevated in nPAP group patients when compared with controls (Figure 1), the difference was significant. $P$ were $0.008,0.002$ and 0.004 , respectively. And there was no significant difference of PAP and IL- 6 in serum between nPAP group and the control group (Figure 1), p were 0.121 and 0.304 , respectively.

In general, the expression of TLR4 mRNA, NF- $\mathrm{kB}$ mRNA in PBMC and of IL-6, TNF- $\alpha$ in serum were significantly elevated in $\mathrm{PAH}$ patients with COPD.

Linear regression analysis of PAP with TLR4/NF-KB and IL- 6 TNF- $\alpha$ of PAH patients with COPD showed that TLR4 was significantly correlated with PAP $(p=0.001)$, both of other pairwise indicators, but the linear positive correlation between IL- 6 with TNF- $\alpha$ was not established $(p=0.170)$, shown in Table I and Figure 2.

Whether the nPAP group or the control group, the strength of association between the indicators was not significant, as shown in Table II.

\section{DISCUSSION}

Toll-like receptors (TLRs) are congenital immunological pathogenic pattern recognition receptors which distribute on the surface of immune cells and play a key role in body defence against biological invasion. They construct a bridge between the natural immune defence and acquired immune system. ${ }^{5}$ TLRs are one of the main groups of pathogen sensors in the innate immune response. They are constituted by an extracellular domain with leucine-rich repeat (LRR), a transmembrane region and a TLR-IL1 domain, which is intracellular. Its extracellular domain is primarily responsible for the identification of pathogen specific ligands and the formation of receptor complexes with other ligands. The intracellular domain contains a domain similar to the IL-1 
receptor (Toll-IL-1 receptor domain, TIR).TIR has the function of homophilic interaction, when TIR binds to the specific adaptor protein, a cascade of signaling cascades is initiated. These specific adaptor proteins contain myeloid differentiation primary response protein 88 (MyD88), MyD88-adaptor-likeprotein (MAL), and so on. ${ }^{6-8}$

As one of the most important members of the TLRs family, TLR4 is discovered the earliest in mammalian TLRs, which belongs to Class I transmembrane proteins. It is widely expressed in many kinds of cells in the human body, mainly distributes in monocytes / macrophages, coronary artery endothelial cells, renal tubular epithelial cells, and peripheral vascular bundle. TLR4 ligands contain endogenous ligands and exogenous ligands. Endogenous ligand is a kind of molecule releasing from injured cells or the decomposition product from extracellular matrix including heat shock protein, high mobility group protein (HMGBL), fibronectin-EDA, low density lipoprotein modified by fetuin-A etc. It does not need external pathogen invasion to activate the natural immune inflammatory response. Exogenous ligands contain lipopolysaccharide (LPS), microbial nucleic acids, etc. ${ }^{9}$

NF-KB is widely present in eukaryotic cells and is a nuclear transcription factor which belongs to the "Rel" family. ${ }^{10} \mathrm{NF}$ $\mathrm{KB}$ is mainly related to the information transmission in the cells and regulates expression of a large number of genes, which are critical for the regulation of apoptosis, viral replication, tumorigenesis, inflammation, and various autoimmune diseases. It contains a Rel homology domain (RHD), which is usually composed of RelA (p65) and p50. This heterodimer and its inhibitory molecules IKB (inhibitor of NF-KB) form a melamine in the cytoplasm in inactivation state. The activation of NF-kB is thought to be part of a stress response. When the upstream signal activates $\mathrm{I-KB}$ in a variety of factors, which include cytokines, inflammatory mediators, pathogens, oxidative stress, NF-KB is activated to enter the nuclear to activate immune response and cell differentiation and other processes. ${ }^{11}$ The NF-KB activation genes mainly contain cytokines (TNF- $\alpha$, IL-1, IL-6, interferon, etc.), adhesion molecules, viral protein growth factors, enzymes and so on. NF-kB plays a unique role in signal transduction, neural plasticity, neural deformation and neural development, immunity, stress response, inflammation and apoptosis.

TLR4/NF-KB signalling pathway is closely related to the antiinflammatory and immune mechanism in recent researches. It plays an important role in the occurrence and development of inflammation. ${ }^{12}$ TLRs signal transduction happens through the dependent and non-dependent pathway of myeloid differentiation factor 88 (MyD88). MyD88 dependent pathway is the classical pathway of signal transduction, the process is that TLR4 combines with its specific ligand in the cell to raise MyD88, and MyD88 gets bridge with its specific adaptor protein $(\mathrm{MAL})$, receives the associated kinase (IRAK) of signal protein IL-1 receptor and get interactions with TNF receptor associated factor 6 (TRAF6), leading to activate the induced kinase of NF-KB, phosphorylation of I$\mathrm{KB}$ kinase, release NF-KB from the melamine and transfer it to the nucleus, and initiate an inflammatory cascade, Leading to the transcription and translation of related genes, and ultimately a large number of inflammatory factors released. $^{13}$

$\mathrm{PAH}$ is a fatal disease with limited therapeutic options. It is pathological state of pulmonary circulation pressure higher than normal. Inflammatory mechanism appears to play a major role in the pathogenesis of various types of human $\mathrm{PAH} .{ }^{14}$ Le et al. believed that inflammation and endothelial dysfunction is a major cause of $\mathrm{PAH} .{ }^{15}$ Tuder $^{16}$ has detected $\mathrm{T}$ Iymphocyte, B lymphocytes, macrophages and other inflammatory cells infiltrating in $\mathrm{PAH}$ patients plexiform lesion of the arterial around. In the process of pulmonary vascular remodelling in $\mathrm{PAH}$, inflammatory cells influx into the vascular wall and the surrounding tissue, high expression of various inflammatory markers is found in lung tissue and circulation. ${ }^{17,18}$ In recent years, the study finds that PAH is often secondary to some systemic inflammatory diseases, such as systemic lupus erythematosus, systemic scleroderma, COPD, and so on. Studies have found that the serum anti-nuclear antibodies, inflammatory cytokines IL-I and IL-6 were highly expressed in patients with idiopathic $\mathrm{PAH}$ (IPAH), while the IL-I receptor antagonists can reduce the degree of $\mathrm{PAH} .{ }^{19}$ Studies suggest that inflammation plays an important role in the pathogenesis of $\mathrm{PAH}$, and reflects the poor clinical outcome..$^{20,21}$

The circulating levels of inflammatory cytokines in $\mathrm{PAH}$ patients increased significantly and inflammatory factors are regulated by NF-KB, which in the downstream of TLR4 signalling pathways is considered to be an important initiating factor of the inflammatory cascade. After activation, NF- $\mathrm{kB}$, initiates and regulates a series of inflammatory factor expression which participate in inflammatory reaction. $^{22}$ As a main upstream receptor of NF-kB, the activation of TLRs may lead to the expression of a large number of NF$\mathrm{kB}$. TLR4 mediates the signal transduction of lipopolysaccharide (LPS) by NF-KB activation pathway, thus it plays an important role in anti-infection in the early stage of pathogen invasion. ${ }^{23}$ In recent years, a large number of studies have shown that TLR4 plays an important role in promoting the synthesis and release of cytokines, trigger inflammation, promoting immune cell membrane surface expression immune molecules, promoting immune cell maturation and function, mediateing systemic immune pathological injury, antiviral infection, regulation of the immune response, and the synergistic effects of the family, etc. ${ }^{24}$

Our research showed that in $\mathrm{PAH}$ patients with COPD, the indicators between PAP with PBMC TLR4, NF-KB and serum IL-6, TNF- $\alpha$ were positively related. It suggests that the 
inflammatory mechanism play an important role in the formation of PAH in patients with COPD, and the degree of inflammation increased with the increase of PAP. In the same patient group, the expression of PBMC TLR4, NF-KB and serum inflammatory factor IL-6, TNF- $\alpha$ were also significant linear positive correlation, suggesting that the TLR4/N$\mathrm{F}-\mathrm{KB}$ signalling pathway, mediates the inflammatory process of PAH. The TLR4/NF-kB signalling pathway, which regulates the inflammatory mechanism, may be able to control the inflammatory process and disease progression of PAH formation in patients with COPD. ${ }^{25}$

As research continues, our cognition will greatly expand about the complexity of the inflammatory immune response in the formation of PAH patients with COPD. Further, it will lay the foundation for finding new targets of drug and gene therapy of PAH with COPD.

\section{CONCLUSION}

Inflammatory mechanisms play an important role in the development of PAH in patients with COPD. TLR4/NF-KB signal transduction pathway is involved in the pathogenesis of PAH, and the expression levels of TLR4/NF-KB may reflect the severity of PAH in patients with COPD.

\section{PATIENTS' CONSENT:}

Informed consents were obtained from all participants.

\section{CONFLICT OF INTEREST:}

Authors declared no conflict of interest.

\section{AUTHORS' CONTRIBUTION:}

ZZ: Led and conceived the project, and authored the manuscript.

MY: Data collection, compiling, statistics and discussion.

SY: Contributed to design articles, collected and analysed data.

BC: Contributed to collect and analyse data.

ZH: Collected and analysed data, literature review and discussion.

CB: Helped perform the analyses with constructive discussions.

\section{REFERENCES}

1. Zeng MY, Tong QY. Anti-inflammation effects of sinomenine on macrophages through suppressing activated tlr $4 / \mathrm{n}$ f-kappab signaling pathway. Curr Med Sci 2020; 40(1): 130-7.

2. Thenappan T, Ormiston ML, Ryan JJ, Archer SL. Pulmonary arterial hypertension: Pathogenesis and clinical manage-ment. BMJ 2018; 360:j5492.

3. Sun SC. The non-canonical NF-kappaB pathway in immunity and inflammation. Nat Rev Immunol 2017; 17(9):545-58.

4. Vogelmeier CF, Criner GJ, Martinez FJ, Anzueto A, Barnes PJ, Bourbeau J, et al. Global Strategy for the diagnosis, management, and prevention of chronic obstructive lung disease 2017 report: GOLD executive summary. Am J Respir Crit Care Med 2017; 195(5):557-82.

5. Tartey S, Takeuchi O. Pathogen recognition and Toll-like receptor targeted therapeutics in innate immune cells. Int
Rev Immunol 2017; 36(2):57-73.

6. Gulati A, Kaur D, Krishna Prasad GVR, Mukhopadhaya A. PRR function of innate immune receptors in recognition of bacteria or bacterial ligands. Adv Exp Med Biol 2018; 1112: 255-80.

7. Anthoney N, Foldi I, Hidalgo A. Toll and toll-like receptor signalling in development. Development 2018; 145(9): $\operatorname{dev} 156018$.

8. Guo S, Nighot M, Al-Sadi R, Alhmoud T, Nighot P, Ma TY. Lipopolysaccharide regulation of intestinal tight junction permeability is mediated by t/r4 signal transduction pathway activation of fak and myd88. J Immunol 2015; 195(10):4999-5010.

9. Mazur-Bialy Al, Pochec E, Zarawski M. Anti-Inflammatory properties of irisin, mediator of physical activity, are connected with tIr4/myd88 signaling pathway activation. Int J Mol Sci 2017; 18(4):701.

10. Mao G, Smyth SS, Morris AJ. Regulation of PLPP3 gene expression by NF-kappaB family transcription factors. J Biol Chem 2019; 294(38):14009-19.

11. Giridharan S, Srinivasan M. Mechanisms of NF-kappaB p65 and strategies for therapeutic manipulation. J Inflamm Res 2018; 11:407-19.

12. Zusso M, Lunardi V, Franceschini D, Pagetta A, Lo R, Stifani $\mathrm{S}$, et al. Ciprofloxacin and levofloxacin attenuate microglia inflammatory response via TLR4/NF-kB pathway. J Neuroinflammation 2019; 16(1):407-19.

13. Guven-Maiorov E, Keskin O, Gursoy A, VanWaes C, Chen Z, Tsai $\mathrm{CJ}$, et al. The architecture of the tir domain signalosome in the toll-like receptor-4 signaling pathway. Sci Rep 2015; 5:13128.

14. Goldenberg NM, Rabinovitch M, Steinberg BE. Inflammatory basis of pulmonary arterial hypertension: Implications for perioperative and critical care medicine. Anesthesiology 2019; 131(4):898-907.

15. Le Hiress M, Tu L, Ricard N, Phan C, Thuillet R, Fadel E, et al. Proinflammatory signature of the dysfunctional endothelium in pulmonary hypertension. role of the macrophage migration inhibitory factor/cd74 complex. Am J Respir Crit Care Med 2015; 192(8):983-97.

16. Tuder RM. How do we measure pathology in PAH (lung and RV) and what does it tell us about the disease. Drug Discov Today 2014; 19(8):1257-63.

17. Ross DJ, Hough G, Hama S, Aboulhosn J, Belperio JA, Saggar $R$, et al. Proinflammatory high-density lipoprotein results from oxidized lipid mediators in the pathogenesis of both idiopathic and associated types of pulmonary arterial hypertension. Pulm Circ 2015; 5(4):640-8.

18. Yang JM, Zhou R, Zhang M, Tan HR, Yu JQ. Betaine attenuates monocrotaline-induced pulmonary arterial hypertension in rats via inhibiting inflammatory response. Molecules 2018; 23(6):1274.

19. Li Y, Li XH, Yu ZX, Cai JJ, Billiar TR, Chen AF, et al. HIV protease inhibitors in pulmonary hypertension: Rationale and design of a pilot trial in idiopathic pulmonary arterial hypertension. Pulm Circ 2015; 5(3):538-46.

20. Harbaum L, Baaske KM, Simon M, Oqueka T, Sinning C, Glatzel A, et al. Exploratory analysis of the neutrophil to 
lymphocyte ratio in patients with pulmonary arterial hypertension. BMC Pulm Med 2017; 17(1):72.

21. Quarck R, Wynants M, Verbeken E, Meyns B, Delcroix M. Contribution of inflammation and impaired angiogenesis to the pathobiology of chronic thromboembolic pulmonary hypertension. Eur Respir J 2015; 46(2):431-43.

22. Li L, Wei C, Kim IK, Janssen-Heininger Y, Gupta S. Inhibition of nuclear factor-kappaB in the lungs prevents mono-crotaline-induced pulmonary hypertension in mice. Hypertension 2014; 63(6):1260-9.

23. Ding RR, Chen W, Guo CY, Liao WT, Yang X, Liao FE, et al.
Dangguishaoyao-San attenuates LPS-induced neuroinflam-mation via the TLRs/NF-kappaB signaling pathway. Biomed Pharmacother 2018; 105:187-94.

24. Garibotto G, Carta A, Picciotto D, Viazzi F, Verzola D. Toll-like receptor-4 signaling mediates inflammation and tissue injury in diabetic nephropathy. J Nephrol 2017; 30(6): 719-27.

25. Ma J, Tian Y, Li J, Zhang L, Wu M, Zhu L, et al. Effect of bufei yishen granules combined with electroacupuncture in rats with chronic obstructive pulmonary disease via the regulation of tlr-4/nf-kappab signaling. Evid Based Complement Alternat Med 2019; 2019:6708645. 